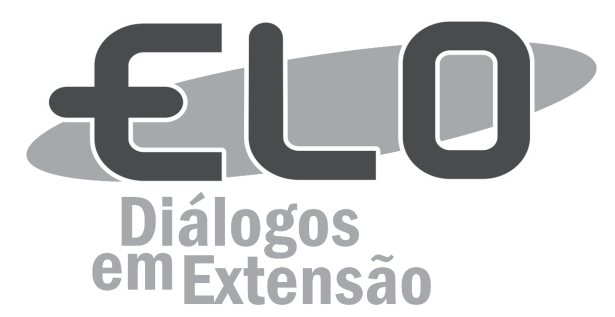

\title{
A indissociabilidade entre ensino, pesquisa e extensão por meio da ACIEPE: Busca, análise e divulgação de informações econômicas do setor agropecuário
}

Estevan Henrique Coelho ${ }^{1}$; Matheus Sleiman da Costa ${ }^{2}$; Marta Cristina Marjotta Maistro ${ }^{3}$; Adriana Estela Sanjuan Montebello ${ }^{4}$

\begin{abstract}
Resumo: As Atividades Curriculares de Integração Ensino, Pesquisa e Extensão (ACIEPE's) são atividades fomentadas pela Pró-Reitoria de Extensão da Universidade Federal de São Carlos, que procuram viabilizar e estimular atividades que envolvam ensino pesquisa e extensão, principal enfoque pedagógico da Instituição. A ACIEPE, projeto direcionado para busca, aná-lise e divulgação de informações econômicas do setor agropecuário, teve por objetivo desen-volver um Boletim Informativo, estimulando os alunos no trabalho de dados e informações, de forma que estes sejam repassados em linguagem simplificada e que se atinja os agentes econômicos em vários níveis. Foram feitos levantamentos e análise de informativos já exis-tentes para basear o desenvolvimento do Boletim Informativo GEAgro, o que resultou na ela-boração de 5 edições de um informativo, sendo estas divulgadas via rede social. A atividade trouxe resultados no sentido de trabalhar com os participantes a indissociabilidade entre Ensi-no, Pesquisa e Extensão, com a perspectiva de continuidade.
\end{abstract}

Palavras-chave: Boletim. Informações. Agropecuário. Economia.

Área Temática: : Educação, Comunicação.

The indivisibility of teaching, research and extension through ACIEPE : search, analysis and dissemination of economic information in the agricultural sector

Abstract: Curricular Activities Education Integration and Research (ACIEPE's) activities are promoted by the Dean of Extension of the Federal University of São Carlos, seek viable and stimulating activities involving education research and extension, which is the main pedagogical approach. The ACIEPE: search, analysis and dissemination of economic information in the agricultural sector aimed to develop a newsletter, encouraging students to work data and information, so that it is passed in simplified language, achieving economic agents at various levels. Were made survey and analysis of existing information on which to base the development of GEAgro Newsletter, which resulted in the preparation of 5 issues an information, which are issued via the social network. The activity brought results in order to work with participants inseparability of Education and Research, with the prospect of continuity.

Keywords: Newsletter. Information. Agricultural. economy.

La indivisibilidad de enseñanza, investigación y extensión a través de ACIEPE : búsqueda, análisis y difusión de información económica en el sector agrícola

Resumen: Actividades Curriculares Integración Educación e Investigación de actividades (de ACIEPE) son promovidos por el Decano de Extensión de la Universidad Federal de San Carlos, buscan actividades viables y estimulantes que implican la investigación y la extensión de la educación, que es el principal enfoque pedagógico.

${ }^{1}$ Discente da Universidade Federal de São Carlos- Campus Araras - SP.

${ }^{2}$ Discente da Universidade Federal de São Carlos- Campus Araras - SP

${ }^{3}$ Docente/Orientadora do projeto - Universidade Federal de São Carlos- Campus Araras - SP. Departamento de Tecnologia Agroindustrial e Socioeconomia Rural - Rod. Anhanguera, km 174. Cep. 13.600970. C. P. 153

${ }^{4}$ Docente - Universidade Federal de São Carlos- Campus Araras - SP 
El ACIEPE: búsqueda, análisis y difusión de información económica en el sector agrícola, al objeto de desarrollar un boletín de noticias, animando a los estudiantes a trabajar los datos y la información, por lo que se pasa en un lenguaje simplificado, el logro de los agentes económicos en los distintos niveles. Se hicieron encuestas y análisis de la información existente sobre la que basar el desarrollo de GEAgro Newsletter, lo que dio lugar a la preparación de un 5 temas de información, que se emiten a través de la red social. La actividad reunió los resultados con el fin de trabajar con los parti-cipantes inseparabilidad de Educación e Investigación, con la perspectiva de continuidad.

Palabras clave: Boletín. Información. Agrícola. economía.

\section{Introdução}

As Atividades Curriculares de Integração Ensino, Pesquisa e Extensão (ACIEPE's) são atividades fomentadas pela Pró-Reitoria de Extensão da Universidade Federal de São Carlos (UFSCar). Por meio dos professores, técnicos e alunos da universidade, essas atividades procuram viabilizar e estimular a interação com diferentes segmentos da sociedade, contribuindo também diretamente na formação profissional, que é o principal enfoque pedagógico da Instituição. (UFSCAR, 2015).

A ACIEPE pode ser desmembrada em ensino, pesquisa e extensão. Como ensino e pesquisa, busca a experimentação de alternativas de solução e encaminhamento de problemas. Como extensão, busca vincular atividades práticas e extraclasse, nas quais o aprendizado se estabelece e é sedimentado. (UFSCAR, 2015).

Tendo em vista os objetivos de uma ACIEPE, no primeiro semestre de 2015, foi ofertado pelo Departamento de Tecnologia Agroindustrial e Socioeconomia Rural (DTAiSER/UFSCar) a ACIEPE: Busca, Análise e Divulgação de Informações Econômicas do Setor Agropecuário. Esta ACIEPE teve como justificativa o fato de que os agentes econômicos envolvidos nesse setor produzem informações e, ao mesmo tempo, necessitam delas para a tomada de decisão. Nesse sentido, vários órgãos, sejam eles de caráter público ou privado, têm compilado e divulgado tais informações.

Em razão da amplitude do universo informativo, torna-se importante ter um olhar crítico sobre o que é divulgado (olhar esse exercido no processo de ensino e aprendizagem), começando, primeiramente, por saber onde procurar; em seguida, avaliar a importância dessa informação para os agentes dos diferentes setores agropecuários (portanto, incentivando a pesquisa) e, por fim, elaborar formas para que a informação seja divulgada (atendendo o caráter extensionista da atividade). Logo, a indissociabilidade entre pesquisa, ensino e extensão fica evidenciada.

Também, no que se refere ao desenvolvimento de atividades de extensão, deve-se ressaltar que, de acordo com o atual Plano Nacional de Educação (PNE), para o período de 2011 a 2020, em sua Meta 12 (elevar a taxa bruta de matrícula na educação superior para $50 \%$ e a taxa líquida para $33 \%$ da população de 18 a 24 anos, assegurando a qualidade da oferta), uma das estratégias para atingir tal meta é assegurar, no mínimo, $10 \%$ do total de créditos curriculares exigidos para a graduação em programas e projetos de extensão universitária (Ministério da Educação, 2016). Nesse sentido, a atividade apresentada neste Relato de Experiência, vem ao encontro da estratégia sugerida para o alcance da Meta 12 do PNE.

\section{Objetivos}

A ACIEPE teve por objetivos:

1. Buscar fontes de informações e dados econômicos voltados para o setor agropecuário;

2. Analisar a pertinência e a relevância de tais informações e dados;

3. Elaborar um Boletim Informativo, com a apresentação dos dados coletados em forma de gráficos e tabelas;

5. Possibilitar, por meio do Boletim Informativo, a divulgação de análises qualitativas dos dados, de uma forma que possa ser compreendida tanto por indivíduos ligados diretamente ao setor agropecuário, como também por aqueles que procuram entender a economia das cadeias produtivas. 


\section{Metodologia}

A ACIEPE foi ofertada na UFSCar, no campus de Ciências Agrárias, na cidade de Araras-SP, pertencente a Mesorregião de Piracicaba e Microrregião de Limeira. O público-alvo foi tanto a comunidade interna como a externa, que são compostas, em sua maioria, por estudantes de agrárias e produtores rurais.

Dada a característica da ACIEPE de proporcionar um espaço de discussão para os temas abordados, com flexibilidade de horário, o cronograma foi elaborado (descritas as etapas a seguir), juntamente aos participantes inscritos, de acordo com o calendário acadêmico e a disponibilidade de salas.

- Cronograma de Atividades da ACIEPE:

\begin{tabular}{lcccc}
\hline \multicolumn{1}{c}{ Atividade/Mês } & \multicolumn{3}{c}{$\mathbf{2 0 1 5}$} \\
\cline { 2 - 5 } & mar & abr & mai jun & jul \\
\hline Levantamento e Análise de informativos disponíveis no mercado. & $X$ & & & \\
Análise e levantamento de informações que possam caracterizar informativos. & $X$ & $X$ & & \\
Desenvolvimento de informativos baseados em ACIEPE's anteriores. & & $X$ & & \\
Desenvolvimento de informativo com novas informações. & & $X$ & $X$ & $X$ \\
Discussão dos resultados e divulgação dos informativos na página do Geagro. & & & $X$ & $X$ \\
\hline
\end{tabular}

Foram feitos levantamentos e análises de informativos já existentes, tais como: o SIFRECA (ESALQLOG, 2014), Boletim do Leite (CEPEA, 2014), $1^{\circ}$ Levantamentos de Safra 2014/2015 (CONAB, 2014).

Concomitantemente, por meio de reuniões do grupo, foram discutidos temas para futuros Boletins Informativos, levando-se em consideração a importância desses do mesmo-para o setor agropecuário e a continuidade da atividade.

Os dados e análises foram apresentados por meio de gráficos e tabelas em arquivos organizados no Power Point e a edição final disponibilizada na página do Grupo de Estudos do Agronegócio (GEAgro), no Facebook.

\section{Resultados e Discussão}

Conforme citado na Metodologia, após os levantamentos e análises de informativos já existentes, o grupo de participantes da atividade de extensão elaborou os informativos. A decisão referente aos temas a serem abordados foi tomada tendo tentelo em vista a importância econômica, de cada tema, para o universo do agronegócio.

Como resultado, foram gerados cinco informativos que procuraram agregar informações relevantes para o setor a que se destinou, sendo que esses foram disponibilizados em formato eletrônico. O primeiro Boletim Informativo, publicado no mês de junho de 2015, tratou do desempenho do agronegócio brasileiro nos anos 2000 e trouxe informações referentes as balanças comerciais do agronegócio com enforque nos três principais complexos: soja, milho e sucroalcooleiro (Figura 1).

O segundo informativo, publicado no mês de Julho de 2015, apresentou dados atualizados da balança comercial do agronegócio e os parceiros comerciais do Brasil, no primeiro semestre de 2015, em comparação ao primeiro semestre de 2014, evidenciando dados de importação e exportação dos parceiros e também de blocos econômicos. (Figura 2)

Ainda, como resultado, os Boletins Informativos de números 3, 4 e 5 foram divulgados ao longo dos meses de agosto, setembro e outubro de 2015, por meio eletrônico. Os temas abordados foram "Vantagens do Produtor no Mercado Físico e de Futuro do Milho"; "Variáveis Econômicas como Peça Chave na Tomada de Decisão de Armazenamento de Soja"; e "Mercado de Equipamentos Agrícolas". Visa-se prosseguir com o projeto de forma constante, garantindo uma periodicidade para a divulgação dos Boletins Informativos.

A principal forma de divulgação ocorreu por meio de redes sociais. Como balanço do alcance das publicações, pode-se considerar a quantidade de pessoas alcançadas: Boletim n.01, 538; Boletim n.02, 872; Boletim n. 03, 508; Boletim n.04, 709 (GEAgro, 2016). O Boletim de n. 05 foi apresentado no V Encontro do GEAgro, com a participação de cerca de 80 pessoas.

A perspectiva de continuidade desta atividade pressupõe a busca por novos meios de divulgação dos Boletins. 

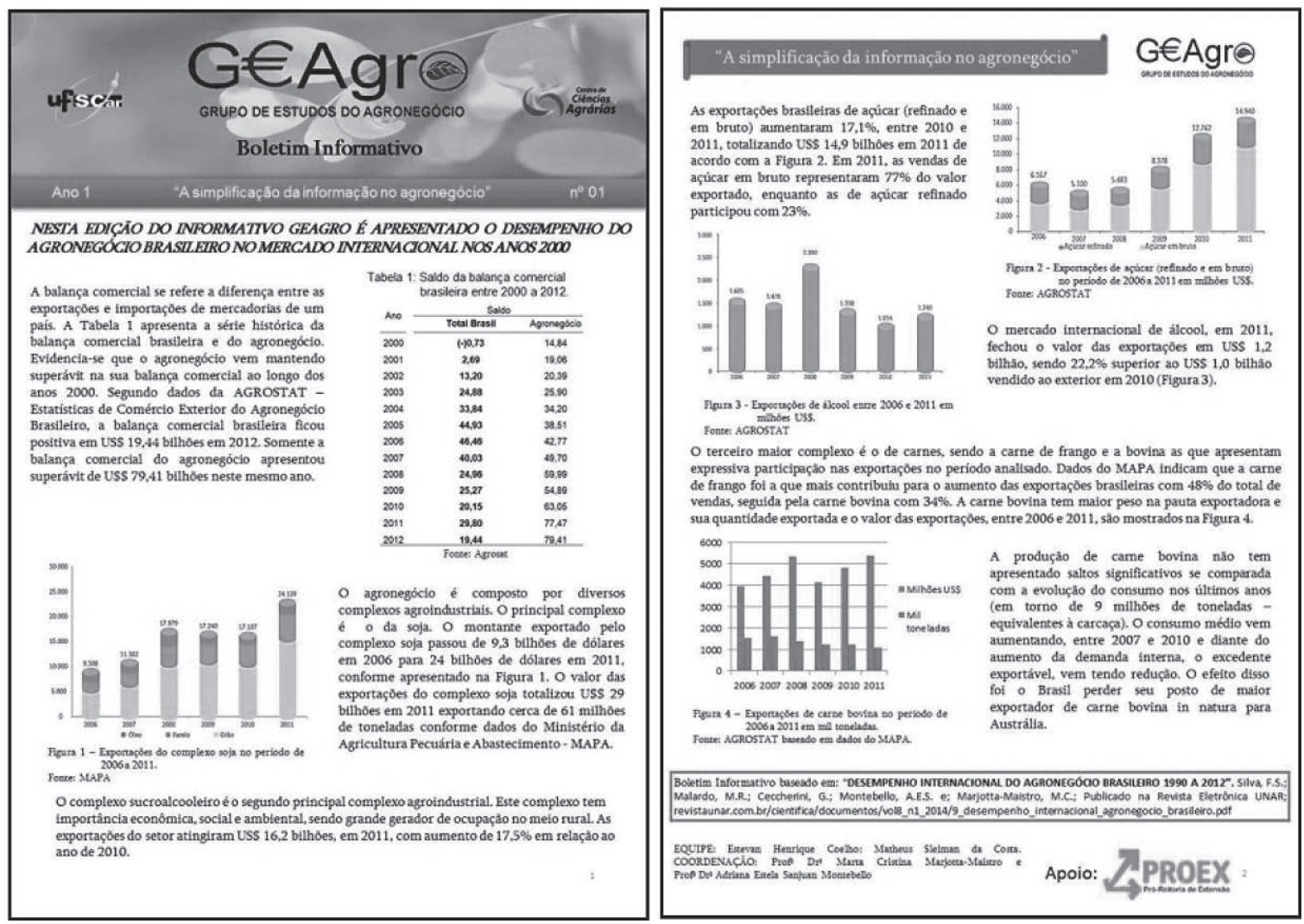

Figura 1 - Informativo 1 - Desempenho do Agronegócio Brasileiro no Mercado Internacional- junho de 2015.

Fonte: Elaborado pelos autores.
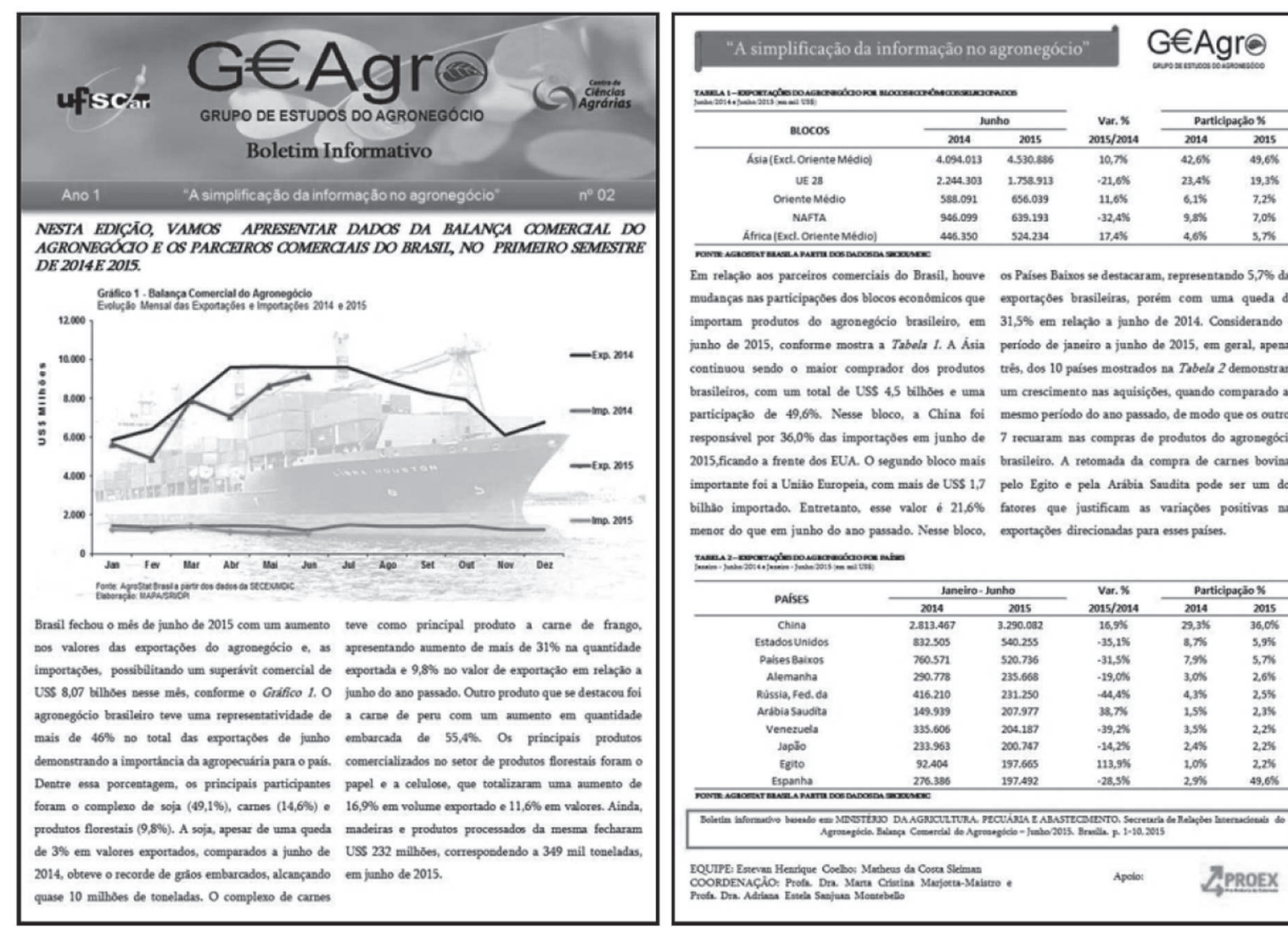

Em relagto aos parceiros comerciais do Brasil, houve os Paises Baixos se destacaram, representando 5,7\% das

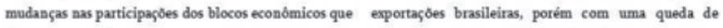
importam produtos do agronegicio brasileiro, em $31,5 \%$ em relapalo a funho de 2014. Considerando o junho de 2015, conforme mostra a Tabela 1. A Ásia periodo de janeiro a junho de 2015, em geral, apenas continuou seddo o maior comprador dos produtos tuts, des 10 parises mostrados na Tabela 2 demonstram brasileiros, com um tocal de USS 4,5 tillhöes e uma um crescimento nas aquisiç̧es, quando comparado ao participaça de 49,6\%. Nesse bloco, a China foi mesmo período do ano passado, de modo que os outros responsivel por 36,0\% das importaģes em junho de 7 recuaram ass compras de produtos do agrobegócio 2015,ficando a frente des EUN. O segundo bloco mais brasileiro, A retomada da compra de carnes bovinas importante foi a Uniaso Europeia, com mais de USS 1,7 pelo Egito e pela Aribia Sundita pode ser um dos bilhào importada. Eatretanto, esse valor $821,6 \%$ fatores que justificam as variaçes positivas nas menor do que em junho do aso passado. Nesse bloco, exportaçoes dirccionadas para esses paises.

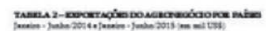

\begin{tabular}{|c|c|c|c|c|c|}
\hline \multirow[b]{2}{*}{ paises } & \multicolumn{2}{|c|}{ Janeiro- Junho } & \multirow{2}{*}{$\begin{array}{c}\text { Var. } \\
2015 / 2014\end{array}$} & \multicolumn{2}{|c|}{ Participaçäo \% } \\
\hline & 2014 & 2015 & & 2014 & 2015 \\
\hline China & 2.813 .467 & 3.290 .032 & $16,9 \%$ & $29.3 \%$ & $30.0 \%$ \\
\hline Estados Unides & 832505 & $500.25 s$ & .35,1\% & $8,7 \%$ & $5,9 \%$ \\
\hline Paises Baixos & 760.571 & 520.736 & $-31.5 \mathrm{~s}$ & 7996 & $5, \pi \%$ \\
\hline Alemanha & 200.778 & 235.668 & -19,0\% & $3,0 \mathrm{x}$ & $2.6 \%$ \\
\hline Russia, Fed.da & 416.210 & 231.250 & $-44,4 \%$ & $4,3 \%$ & $2.5 \%$ \\
\hline Artbia Savitata & 149.939 & $207.9 n$ & $38,7 \times$ & $1.5 \%$ & 2.36 \\
\hline venezuels & 335.606 & 204.187 & -39,2\% & 3.5\% & 2,25 \\
\hline Japto & 23.963 & 200.747 & $-10,25$ & $2,4 \%$ & 2.256 \\
\hline Evito & 92404 & 197.665 & 113,956 & $1,0 \%$ & 2.266 \\
\hline \multicolumn{5}{|c|}{ 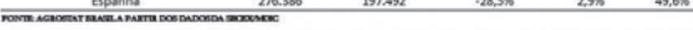 } & \\
\hline \multicolumn{6}{|c|}{ 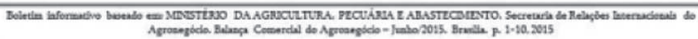 } \\
\hline \multicolumn{3}{|c|}{ 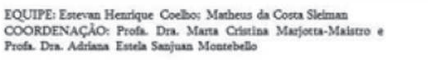 } & Apolo: & \multicolumn{2}{|c|}{$\angle \mathrm{PROEX}$} \\
\hline
\end{tabular}

Figura 2: Informativo 2 - Balança Comercial do Agronegócio e os Parceiros Comerciais do Brasil, no primeiro semestre de 2014 e 2015. Julho de 2015.

Fonte: Elaborado pelos autores. 


\section{Conclusão}

A temática discutida e os resultados alcançados por meio desta ACIEPE subsidiam algumas reflexões acerca da relevância acadêmica e social da atividade.

A relevância acadêmica se verificou no fato de que a ACIEPE teve como objeto de pesquisa o setor agropecuário, focando discussões que permeiam as decisões deste setor. -Nesse sentido, as informações econômicas levantadas são de suma importância para diagnosticar os gargalos nas áreas relacionadas a esses setores, como a comercialização e, se possível, propor soluções para os mesmos.

Em termos de relevância social, o fato de saber onde buscar, divulgar e realizar uma análise crítica das informações econômicas, propicia melhores subsídios para os tomadores de decisões no setor agropecuário. Além disso, o público, em geral, tem a oportunidade de apreender sobre questões que, de alguma forma, permeiam seu dia a dia, dada a percepção de que assuntos relativos à economia tendem a subsidiar decisões de produção e consumo. Vale ressaltar que, pela forma como foi feita a divulgação - via redes sociais - alcançou um número expressivo de pessoas.

Com relação aos objetivos, a ACIEPE serviu para os alunos de aprendizado de como buscar fontes de informações e dados econômicos do setor agropecuário, analisando a pertinência dos dados para a elaboração do boletim, que foi a principal ferramenta para a divulgação dos resultados da atividade.

A ACIEPE - Busca, Análise e Divulgação de Informações Econômicas do Setor Agropecuário, trouxe resultados no sentido de trabalhar com os participantes a indissociabilidade entre Ensino, Pesquisa e Extensão, atingindo os objetivos da atividade, com a perspectiva de a atividade ter continuidade, reforçando ainda mais a importância do mesmo para a comunidade, tanto acadêmica quanto externa.

\section{Fontes de Financiamento}

Pró-Reitoria de Extensão Universitária da Universidade Federal de São Carlos. (ProEx/UFSCar)

\section{Agradecimento}

Pró-Reitoria de Extensão Universitária da Universidade Federal de São Carlos. (ProEx/UFSCar)

\section{Referências Bibliográficas}

CONAB - Companhia Nacional de Abastecimento - $1^{\circ}$ Levantamentos de Safra 2014/2015. Disponível em: <http://www.conab.gov.br/OlalaCMS/uploads/arquivos/14_10_23_10_20_02

_boletim_graos_outubro_2014.pdf>. Acesso em 28 mar. 2015.

CEPEA - CENTRO DE ESTUDOS AVANÇADOS EM ECONOMIA APLICADA - ESALQ/USP. Boletim do Leite, 228. Disponível em: <http://cepea.esalq.usp.br/leite/boletim/228.pdf>. Acesso em 28 mar. 2015.

ESALQ-Log - Grupo de Pesquisa e Extensão em Logística Agroindustrial da Escola Superior de Agricultura Luiz de Queiroz. SIFRECA No 4. 2014. Disponível em: <http://esalqlog.esalq.usp.br/ sifreca/etanol-dezembro2014-no-04/> Acesso em: 25 mar. 2015.

GEAGRO - GRUPO DE ESTUDOS DO AGRONEGÓCIO. Disponível em: < www.facebook.com/ GEAgro>. Acesso em 12 ago. de 2016.

HEINZMANN, Clara et.al. Adoção de sistemas de informação como estratégia competitiva nas grandes empresas do setor do agronegócio da região oeste do estado do Paraná. In: CONGRESSO LUSOBRASILEIRO DE TECNOLOGIAS DE INFORMAÇÃO E COMUNICAÇÃO NA AGRO-PECUÁRIA, 1,2004, Portugal. Disponível em: < www.agriculturadigital.org/agritic_2004/congresso/E-business_MBusiness_/Adocao_SI_Empresas_Parana.pdf>. Acesso em: 28 de mar. 2015.

MAPA - Ministério da Agricultura, Pecuária e Abastecimento. Disponível em: $<$ www.agricultura.gov.br/politica-agricola/publicacoes/informativo-de-economia-agricola >. Acesso em: 28 de mar. 2015. 
MINISTÉRIO DA EDUCAÇÃO. Projeto de Lei do Plano Nacional de Educação. Disponível em: <http:/ / portal.mec.gov.br/index.php?option=com_docman\&view $=$ download\&alias=7116-pl-pne-20112020\&Itemid=30192>. Acesso em: 15 ago. 2016.

SILVA, ANA PAULA da. Da conversa na praça ao via satélite: a busca por informação agropecuária. Dissertação (Mestrado) - Escola de Comunicações e Artes/USP, 2005. 113p. Disponível em: <www.cepea.esalq.usp.br>. Acesso em: 28 de mar. 2015.

UFSCAR - UNIVERSIDADE FEDERAL DE SÃO CARLOS. Atividade Curricular de Integração Ensino, Pesquisa e Extensão (ACIEPE). Disponível em: <www.ufscar.br/aciepes> . Acesso em: 05 ago. 2015

Recebido para publicação em 25/03/2016 e aprovado em 08/08/2016.

\section{Notas}

*Este trabalho foi apresentado no $2^{\circ}$ Congresso de Extensão da Associação das Universidades do Grupo de Montevidéu, realizado na Unicamp, no período de 09 a 12 de outubro de 2015. 